\title{
The NuGO proof of principle study package: a collaborative research effort of the European Nutrigenomics Organisation
}

\author{
Michela Baccini - Eva-Maria Bachmaier • Annibale Biggeri · Mark V. Boekschoten · Freek G. Bouwman • \\ Lorraine Brennan · Robert Caesar · Saverio Cinti - Susan L. Coort · Katie Crosley • Hannelore Daniel · \\ Christian A. Drevon · Susan Duthie · Lars Eijssen · Ruan M. Elliott · Marjan van Erk · Chris Evelo • \\ Mike Gibney · Carolin Heim · Graham W. Horgan · Ian T. Johnson · Thomas Kelder · Robert Kleemann • \\ Teake Kooistra · Martijn P. van Iersel • Edwin C. Mariman • Claus Mayer • Gerard McLoughlin • \\ Michael Müller · Francis Mulholland • Ben van Ommen · Abigael C. Polley • Estelle Pujos-Guillot • \\ Isabel Rubio-Aliaga - Helen M. Roche $\cdot$ Baukje de Roos • Manuela Sailer • Giulia Tonini $\cdot$ Lynda M. Williams \\ Nicole de Wit · For the NuGO PPS Team
}

Received: 8 October 2008/ Accepted: 12 November 2008/Published online: 26 November 2008

(C) The Author(s) 2008. This article is published with open access at Springerlink.com

\section{Introduction}

The European Nutrigenomics Organisation (NuGO; www.nugo.org) was established in 2004 and is funded within the Sixth Framework Programme of the European Commission as a network of excellence [4]. The core partners forming $\mathrm{NuGO}$ are 23 research units based at universities and research centers. The key objectives of

Authors are arranged in alphabetical order.

M. Baccini · A. Biggeri - G. Tonini

Department of Statistics, University of Florence, Florence, Italy

E.-M. Bachmaier · K. Crosley · S. Duthie · B. de Roos ·

L. M. Williams

University of Aberdeen, Rowett Institute of Nutrition

and Health, Aberdeen, UK

M. V. Boekschoten · M. Müller · N. de Wit

Division of Human Nutrition, Wageningen University,

Wageningen, The Netherlands

M. V. Boekschoten · M. Müller

Nutrigenomics Consortium, TI Food and Nutrition, Wageningen,

The Netherlands

F. G. Bouwman · E. C. Mariman

Department of Human Biology, Maastricht University,

Maastricht, The Netherlands

L. Brennan · G. McLoughlin

University College Dublin School of Agriculture, Food Science and Veterinary Medicine, Dublin, Republic of Ireland

R. Caesar · C. A. Drevon

Department of Nutrition, Institute of Basic Medical Sciences,

University of Oslo, Oslo, Norway
NuGO are: (a) the promotion of molecular nutrition research in Europe; (b) the development and promotion of mechanistic research in nutrition, food and health research by application of omics technologies; and (c) to develop joint research programs. The NuGO Proof of Principle Study package (NuGO PPS) started in 2007 and is one of the collaborative research initiatives within NuGO. The main goals of the NuGO PPS are to: (a) assess the advantages and the limitations of omics applications in nutritional studies; (b) prove that systems biology

S. Cinti

Institute of Normal Human Morphology,

University of Ancona, Ancona, Italy

S. L. Coort - L. Eijssen - C. Evelo - T. Kelder - M. P. van Iersel Department of Bioinformatics, BiGCaT,

Maastricht University, Maastricht,

The Netherlands

H. Daniel $(\varangle) \cdot$ C. Heim · I. Rubio-Aliaga $(\varangle) \cdot$ M. Sailer Department of Nutrition Physiology,

Technische Universität München, 85350 Freising-Weihenstephan, Germany

e-mail: daniel@wzw.tum.de

I. Rubio-Aliaga

e-mail: rubio@wzw.tum.de

R. M. Elliott - I. T. Johnson · F. Mulholland · A. C. Polley Institute of Food Research, Norwich Research Park, Norwich, UK

M. van Erk · B. van Ommen

Department of Physiological Genomics,

TNO-Quality of Life, Zeist, The Netherlands 
approaches can provide a better description of the health/ disease status of an organism; (c) prove NuGO's ability as a network of excellence to analyze complex data sets obtained from a federated series of studies, performed at many partner sites, analyzed in different omics platforms in combination with statistics and bioinformatics in an integrative way; and finally (d) optimize the convergence in data sharing by implementing a data integration platform.

The organizational structure of the NuGO PPS comprises a core team formed by six leading scientists (C.A.D., R.M.E., M.G., R.K., M.M., B.v.O.) who defined the general outlines of the NuGO PPS, a coordinator team (H.D., I.R.-A.), three main centers where the experimental studies were performed and 12 centers where samples are analyzed in a close relationship between "wet lab" and "dry-lab" scientists (Fig. 1). Moreover, there is a study that links NuGO to another Framework 6 Integrated Project, LIPGENE (www.ucd.ie/lipgene), which is an integrated project on the theme of the metabolic syndrome.

The experimental research platform is based on three mouse feeding trials, PPS1, PPS2, PPS3, and a human volunteer trial referred to as the human PPS (both animal and human studies are approved by their respective local ethics committees). All studies are based on an energy challenge and the acute and chronic effects on substrate oxidation and inflammation status in addition to the long term alterations and stability of energy homeostasis. The mouse studies address the effects of time (PPS1) or metabolic challenges (PPS2) or dietary fat content (PPS3) on the development of insulin resistance and the metabolic consequences which are assessed by comprehensive phenotyping. Analysis takes into account the type of diet, the time-dependency of changes and organ-specificity of effects well as the ability of an organism to cope with

\footnotetext{
M. Gibney

University College Dublin, Dublin,

Republic of Ireland
}

G. W. Horgan - C. Mayer

Department of Biomathematics and Statistics Scotland,

University of Aberdeen, Rowett Institute of Nutrition

and Health, Aberdeen, UK

R. Kleemann · T. Kooistra

Department of Vascular and Metabolic Diseases, TNO-Quality of Life, Leiden, The Netherlands

\section{E. Pujos-Guillot}

Unité de Nutrition Humaine, Institut National de la Recherche

Agronomique, St-Genès-Champanelle, France

H. M. Roche

Nutrigenomics Research Group, UCD Conway Institute,

University College Dublin, Dublin, Republic of Ireland metabolic dietary stress (bolus of glucose). The human study is designed to assess the biological intra- and interindividual variation of a huge spectrum of molecular, metabolic and clinical parameters employing the different omics technologies and to assess the scale and patterns of alterations elicited by a simple nutritional intervention-a $36 \mathrm{~h}$ fasting period.

\section{PPS1: development of insulin resistance on a high fat diet in $\mathrm{ApoE}^{* 3 \text { 3Leiden mice }}$}

The ApoE*3Leiden transgenic mouse is highly susceptible to diet-induced hyperlipoproteinemia and has been established as a valid murine atherosclerosis models mimicking many hallmarks of human metabolism related to diabetes and atherosclerosis [8].In ApoE*3Leiden transgenic mice, the lipid metabolism is humanized (i.e., a more human-like lipoprotein profile). These humanized mice also can serve as a model of insulin resistance. The mouse study was designed and executed by T.K. and R.K., at TNO-Leiden (The Netherlands) including microarray analysis of organs relevant for insulin resistance (liver, adipose tissue, muscle). Parts of the study were donated to NuGO and biosamples are being further analyzed within the NuGO PPS1 research community. The prime goal of the study is to monitor, with high time-resolution, the development of diet-induced obesity (DIO) and insulin resistance. To do so, genome-wide transcriptomic datasets for the course of the development of insulin resistance in liver, white adipose tissue and muscle were generated. Within PPS1, the development of hepatic steatosis is analyzed which includes the development of a (subacute) inflammatory response. Insulin resistance was achieved in the ApoE*3Leiden mice by feeding animals a diet based on $45 \%$ fat by energy (beef tallow) over 12 weeks. Insulin resistance was established in the animals by performing a glucose tolerance test 3 day prior to sacrifice (and parallel hyperinsulinemic-euglycemic clamp analyses in separate mice). Tissue samples were collected at $0,1,6,9$ and 12 weeks (sacrifice of $n=15$ animals per time point) and plasma samples were taken in each animal at regular intervals to be able to analyze intra-individual changes over time. Three different adipose tissue depots (mesenteric, abdominal subcutaneous and epididymal) are being analyzed by transcriptomics, histology and immunochemistry to assess the response of the different depots to insulin resistance and to obtain potential new biomarkers of adipose tissue insulin resistance. These analyses will be performed taking into consideration that the mesenteric adipose tissue can be markedly contaminated by pancreatic tissue in the proximal part of the mesenteric adipose depot [2]. Pancreatic contamination of the mesenteric adipose 
Fig. 1 Organizational structure of the NuGO Proof of Principle Study Package. *Study donated by TNO-Quality of Life. TNOQuality of Life, The

Netherlands; Wageningen University, The Netherlands; University of Aberdeen, Rowett Institute of Nutrition and Health (UoA), UK; University of Oslo, Norway; Ancona University, Italy; Maastricht University, The Netherlands; University College Dublin (UCD), Ireland; Institut National de la Recherche Agronomique (INRA), France; Institute of Food Research (IFR), UK;

Technische Universität München (TUM), Germany; Firenze University, Italy

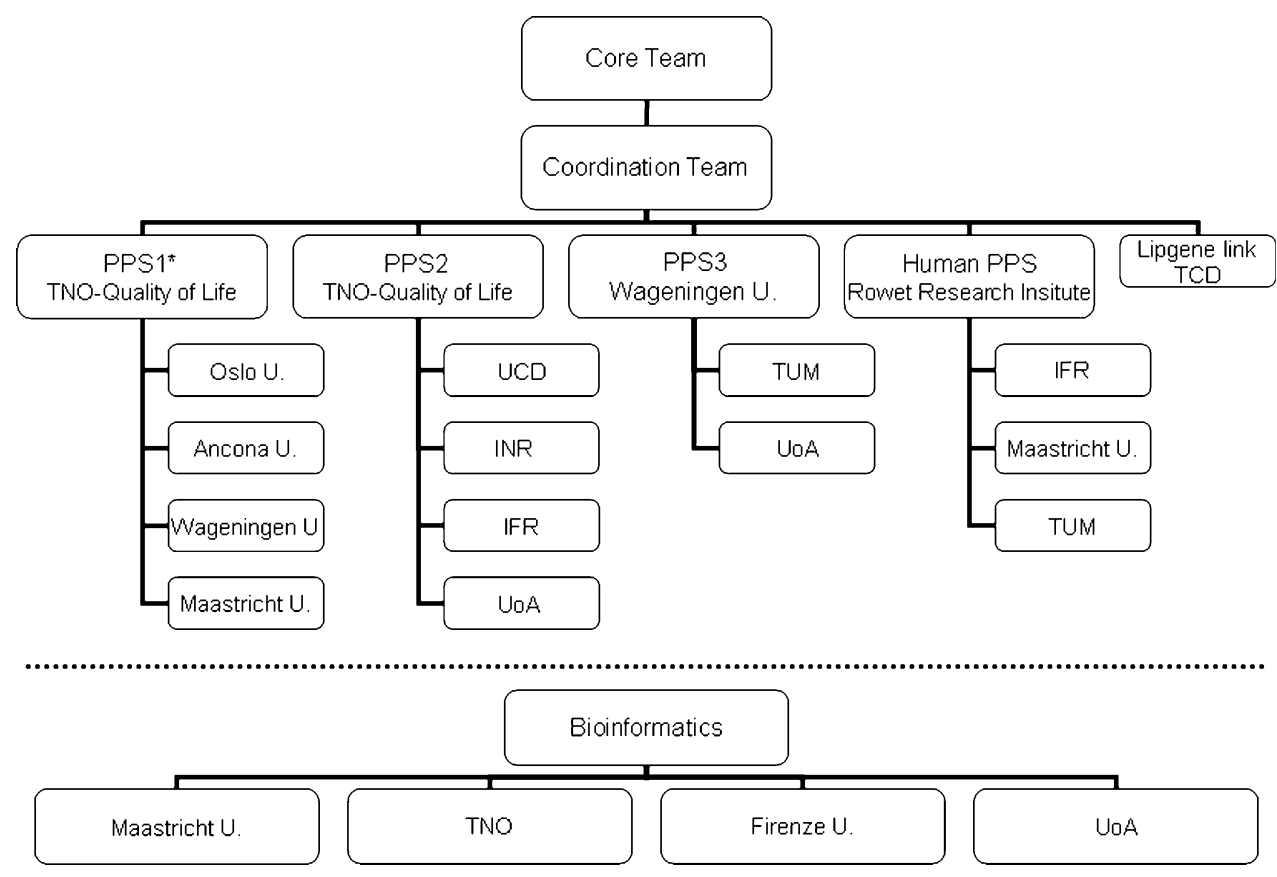

tissue can be minimized by discarding the proximal part of the mesenterium located close to the pancreas. The liver is being analyzed by transcriptomics, targeted proteomics as well as histology and immunochemistry. In addition, inflammation markers (for review: [5, 6]) are monitored in liver and plasma to follow the progression of insulin resistance and hepatic steatosis. Moreover, collaborations have been established with other groups within NuGO to analyze the impact of this metabolic challenge on colonic tissues (G. Elliott and L. Lund, Institute of Food Research, Norwich, UK).

\section{PPS2: evaluation of the acute whole-body response to glucose stress in $\mathrm{C57BL} / 6 \mathrm{~J}$ mice}

PPS2 was designed to compare the whole-body response to a metabolic stressor (glucose bolus) between healthy and insulin resistant mice. Mice with an isogenic background (C57BL/6J mice) were fed diets for 12 weeks containing either 10 or $45 \%$ fat by energy (mainly palm oil) after 2 weeks of adaptation to laboratory conditions with a control diet. Plasma was collected throughout the 12 weeks of feeding to monitor the development of insulin resistance establishing that animals on the high fat diet had developed insulin resistance after 3 months. Healthy and insulin resistant animals were subjected to a glucose tolerance test at the end of the feeding trial followed by comprehensive phenotyping. The core of the study was performed at TNO (coordinated by R.K. and T.K.). Serum, liver and selected tissues like muscle and adipose tissue were removed $0 \mathrm{~min}$, $40 \mathrm{~min}, 2,18$ and $48 \mathrm{~h}$ after the glucose challenge. Glucose was injected via the i.p. route at 1 p.m. in all groups and animals had free access to feed during the challenge (no fasting). The liver will be analyzed by transcriptomics, and by proteomic and metabolomic approaches. Metabolomic analysis is also being performed in muscle, another tissue affected by insulin resistance, whilst serum is being analyzed by both proteomics and metabolomics. Moreover, the collaborations mentioned in PPS1 have been established here also to analyze the impact of this metabolic challenge on colonic tissues.

\section{PPS3: dose-dependent effects of dietary fat on the organ-specific response in C57BL/6J mice}

Prime aim of this study is to generate robust omics data sets for the effects of diets comprised of increasing fat contents on the organism. The core study was performed at Wageningen University (coordinated by M.M. and M.V.B.). C57BL/6J animals were fed diets with 10, 20, 30 and $45 \%$ energy from fat for 4 weeks (after 2 weeks adaptation to a control diet). Transcriptome analysis is being performed in liver, small intestine, adipose tissue and muscle on samples obtained after 0,1 and 4 weeks of feeding. Moreover, proteomic analysis of liver and muscle tissue is being carried out on the same samples to enable direct comparison with the transcriptomic data. Pancreatic tissue is analyzed at the proteomic level due to its importance in insulin secretion. And in collaborations with other $\mathrm{NuGO}$ groups colonic (D. Haller and T. Werner, TUM) and brown adipose tissue (M. Klingenspor and T. Fromme, TUM) are also being analyzed. Since not only glucose and lipid metabolism but 
also amino acid metabolism (5) are insulin-dependent and change during a decline of tissue insulin sensitivity, another aim of PPS 3 is to assess whether changes in plasma amino acid levels or patterns can serve as biomarkers of insulin resistance. LC-MS/MS will be applied to quantify 45 amino acids and derivatives in plasma samples collected every second week from animals fed the 10 or the $45 \%$ energy from fat diets over the 12-week period. Another study arm embedded into PPS3 is comprised of a leptin challenge (coordinated by B.deR. and L.M.W.). In this study we perform transcriptomic analysis of the hypothalamus, proteomic analysis histochemical analysis of hepatic cells in mice fed 10 or $45 \%$ energy from fat for 1 or 4 weeks, with or without an acute leptin challenge. This will allow us to assess, at the tissue level, changes in leptin sensitivity and alterations that are central to satiety and metabolic control $[1,5]$. Such changes will be related to plasma leptin and glucose levels.

\section{Human PPS: an intervention study}

The human PPS represents a continuation and extension of an established collaborative effort within NuGO based on the PlasmaPlus Study in which four centers validated and optimized proteome analysis from blood samples for biomarker discovery [3]. Main aims of the human PPS is to (1) assess the biological intra-and inter-individual variance of a huge spectrum of molecular, metabolic and clinical parameters employing the different omics technologies at baseline and after a $36 \mathrm{~h}$ fasting period, (2) identify common and unique changes in these parameters in response to fasting, and 3) determine the most comprehensive and sensitive omics approaches to detect and quantify the effects of a nutritional fasting challenge in humans. The human PPS enrolled ten healthy volunteers that were asked to come to the Human Nutrition Unit in Aberdeen on four different days of the week during four weeks after an overnight fast to provide a blood sample (for the isolation of plasma, platelets and PBMC), and a saliva and a morning urine sample to assess intra- and interindividual variations in baseline levels. On the fourth sampling day, volunteers were fasted for an additional $24 \mathrm{~h}$ (total of $36 \mathrm{~h}$ ) and again plasma, saliva and urine samples as well as circulating blood cells were collected for transcriptome, proteome and metabolome analysis. The core study was performed at the University of Aberdeen (coordinated by B.deR. and S.J.D.). All samples are being submitted to comprehensive profiling employing all omics technologies. Biological variability and fasting responses are being monitored in plasma, platelets, peripheral blood mononuclear cell (PBMC), urine and saliva by proteomics based on 2D-gel based platforms coupled to mass spectrometry for protein identification. Alterations in the PBMC transcriptome are being analyzed by employing the Affymetrix microarrays specifically designed for NuGO, and plasma and urine sample metabolite profiling is achieved based on NMR, GC-MS, GC-MS/MS and LC-MS/MS platforms comprising non-targeted and targeted approaches. Moreover, a panel of 89 hormones, cytokines and chemokines and metabolic factors was analyzed on a commercial targeted profiling platform (RulesBasedMedicine, www. rulesbasedmedicine.com). In this unique combination of nutrigenomics tools this study should provide the most comprehensive metabolic phenotyping possible in human volunteers with minimal invasive methods for obtaining appropriate biosamples.

\section{Bioinformatic studies}

All data generated in the four experimental studies are being collected and analyzed using a central data warehouse represented by the NuGO "black box system" also comprising a NuGO-wide intranet platform for data processing and analysis. A group of bioinformatic experts (coordinated by C.E) is responsible for the analysis of the data by different bioinformatic and biostatistics approaches. The studies are focused on the statistical modeling of the time-courses and organ-specific responses to the dietary challenges with a particular focus on the transcript profiling data sets. A second series of analysis uses the time course results and targets integration of transcriptomic, metabolomic and proteomic data by multivariate analyses and at the pathway level by using, for example, PathVisio [7]. In this issue Coort S. L. et al. and Baccini M. et al. describe some of the biostatistical and bioinformatic approaches applied to the different study sections with the NuGO PPS.

\section{Standardization and integration}

The NuGO PPS package is the outcome of a long process of discussions between the nutrigenomics experts in the network. Apart from the scientific innovation and excellence mentioned previously, a prime prerequisite was that all partners submitting proposals to join the PPS trial could collaborate within a virtual research infrastructure. All participants had to agree on the highest level of standardization of the methodologies used and on standardized data storage and data sharing policies. In fact, this study was intended also as a proof of principle for the established NuGO research infrastructure. The experimental designs of two of the three mouse studies were harmonized by defining animals, age, gender, diets, fasting periods and the 
workflow in obtaining and processing the biosamples. Shared analytical platforms such as the NuGO Affymetrix gene chip, a common hybridization service as well as the stream-lined data analysis should ensure the highest degree of harmonized data generation and standardization. Also, the NuGO PPS profits from the infrastructure already created in the previous years within NuGO such as the array pipeline and the NuGO Black Box Project which is formed by a federated network of computer servers installed at all partners, allowing standardized storage, sharing and processing of all data generated within the PPS (Evelo et al. in press).

\section{Conclusion}

Here we present the NuGO Proof of Principle Study package to the science community. This effort represents one of the very few coordinated interdisciplinary and multicenter research initiatives in the nutrigenomics field. The aims of the studies are to (1) improve understand of the time-lines for the development of insulin resistance in different organs under feeding regimens based on high fat diets, (2) take the omics technologies to proof and (3) take $\mathrm{NuGO}$ to proof as a network that is generating an "added value" by sharing technology, data, knowledge and expertise. In this respect not only mice and human volunteers are challenged but also the entire science community under the NuGO (p)roof.

Acknowledgments This project is funded by the Nutrigenomics Organisation, EC funded Network of Excellence, grant nr.FOOD2004-506360.

Open Access This article is distributed under the terms of the Creative Commons Attribution Noncommercial License which permits any noncommercial use, distribution, and reproduction in any medium, provided the original author(s) and source are credited.

\section{References}

1. Angulo P, Lindor KD (2002) Non-alcoholic fatty liver disease. J Gastroenterol Hepatol 17(Suppl):S186-S190

2. Caesar R, Drevon CA (2008) Pancreatic contamination of mesenteric adipose tissue samples can be avoided by adjusted dissection procedures. J Lipid Res 49:1588-1594

3. de Roos B, Duthie SJ, Polley AC, Mulholland F, Bouwman FG, Heim C, Rucklidge GJ, Johnson IT, Mariman EC, Daniel H, Elliott RM (2008) Proteomic methodological recommendations for studies involving human plasma, platelets, and peripheral blood mononuclear cells. J Proteome Res 7:2280-2290

4. Kaput J, Ordovas JM, Ferguson L, van Ommen B, Rodriguez RL, Allen L, Ames BN, Dawson K, German B, Krauss R, Malyj W, Archer MC, Barnes S, Bartholomew A, Birk R, van Bladeren P, Bradford KJ, Brown KH, Caetano R, Castle D, Chadwick R, Clarke S, Clement K, Cooney CA, Corella D, Manica da Cruz IB, Daniel H, Duster T, Ebbesson SO, Elliott R, Fairweather-Tait S, Felton J, Fenech M, Finley JW, Fogg-Johnson N, Gill-Garrison R, Gibney MJ, Gillies PJ, Gustafsson JA, Hartman IV JL, He L, Hwang JK, Jais JP, Jang Y, Joost H, Junien C, Kanter M, Kibbe WA, Koletzko B, Korf BR, Kornman K, Krempin DW, Langin D, Lauren DR, Ho Lee J, Leveille GA, Lin SJ, Mathers J, Mayne M, McNabb W, Milner JA, Morgan P, Muller M, Nikolsky Y, van der Ouderaa F, Park T, Pensel N, Perez-Jimenez F, Poutanen K, Roberts M, Saris WH, Schuster G, Shelling AN, Simopoulos AP, Southon S, Tai ES, Towne B, Trayhurn P, Uauy R, Visek WJ, Warden C, Weiss R, Wiencke J, Winkler J, Wolff GL, ZhaoWilson X, Zucker JD (2005) The case for strategic international alliances to harness nutritional genomics for public and personal health. Br J Nutr 94:623-632

5. Pickup JC, Crook MA (1998) Is type II diabetes mellitus a disease of the innate immune system? Diabetologia 41:1241-1248

6. Tataranni PA, Ortega E (2005) A burning question: does an adipokine-induced activation of the immune system mediate the effect of overnutrition on type 2 diabetes? Diabetes 54:917-927

7. van Iersel MP, Kelder T, Pico AR, Hanspers K, Coort S, Conklin BR, Evelo C (2008) Presenting and exploring biological pathways with PathVisio. BMC Bioinformatics 9:399

8. Zadelaar S, Kleemann R, Verschuren L, der Weij J, van der Hoorn J, Princen HM, Kooistra T (2007) Mouse models for atherosclerosis and pharmaceutical modifiers. Arterioscler Thromb Vasc Biol 27:1706-1721 